\title{
Energy Efficiency and SINR Maximization Beamformers for Cognitive Radio Utilizing Sensing Information
}

\author{
AbdulRahman Alabbasi, Zouheir Rezki, \\ Basem Shihada \\ CEMSE Division, King Abdullah University of Science and Technology \\ Email: \{abdulrahman.alabbasi, zouheir.rezki, basem.shihada\}@kaust.edu.sa
}

\begin{abstract}
In this paper we consider a cognitive radio multi-input multi-output environment in which we adapt our beamformer to maximize both energy efficiency (EE) and signal to interference plus noise ratio (SINR) metrics. Our design considers an underlaying communication using adaptive beamforming schemes combined with sensing information to achieve an optimal energy efficient systems. The proposed schemes maximize EE and SINR metrics subject to cognitive radio and quality of service constraints. Since the optimization of EE problem is not a convex problem, we transform it into a standard semi-definite programming (SDP) form to guarantee that the optimal solutions are global. Analytical solution is provided for one scheme, while the other scheme is left in a standard SDP form. Selected numerical results are used to show that both of the proposed schemes, with the sensing information, outperform the same schemes without the sensing information.

Index Terms-Cognitive radio, spectrum sharing, energy efficiency, spectrum sensing, resource allocation, beamforming, MIMO.
\end{abstract}

\section{INTRODUCTION}

Green communication is a major contributor to the global greenness. Therefore, we note the high demand on reducing the mobile phones power consumption while achieving high throughput. It is found that the radio's power consumption reaches up to $50 \%$ of the mobile device's battery power consumption [1]. One of the green communication potential candidates is cognitive radio (CR) and its communication layers [2]. There are many proposals pursuing the greenness in CR technology [2]. CR has been proposed to overcome the inefficient use of frequency spectrum and its resulting scarcity. In $\mathrm{CR}$, secondary users (SUs) may share the bands of the primary users (PUs) through various schemes. Combining the knowledge of PU's existence into the SU's transmission scheme results in an improved performance [1].

Researchers have been considering optimal energy efficient systems by minimizing the system energy while preserving its quality of service (QoS) parameters, such as rate, delay, etc. However, it has been indicated in [3] that optimizing the energy per goodbits (EPG) yields to an improved result in terms of energy efficiency. Authors in [4] have tackled the problem of maximizing the energy efficiency (EE), which is the inverse of EPG, in multi-input multi-output (MIMO) multi-user environment. Recent development of MIMO beamforming techniques create a new dimension for co-channel users to coexist without causing severe interference to each others. In conventional methods, beamforming is obtained by either maximizing the signal to interference plus noise ratio (SINR), or minimizing the transmission energy subject to a minimum SINR constraint. Authors in [5] formulate the SU beamforming problem as a non-convex optimization problem, under the assumption that the CSI on all links is perfectly known to the SUs.

In this paper, we maximize EE and SINR metrics under spectrum sharing MIMO environment utilizing sensing information about PU. The SU's SINR maximization scheme (scheme 1) is constrained with a certain peak power and interference threshold towards the PU while taking into consideration the sensing information. The SU's EE maximization scheme is constrained with a minimum rate constraint in addition to the constraints mentioned in scheme 1 , while utilizing the sensing information. We assume the availability of a spectrum sensor at the SU. That periodically collects real-time information about PU signals in the surrounding environment. This sensing information is obtained by a blind multi-antenna combining scheme (explained in Section II-B). In our framework, we assume that the SU does not have knowledge of the secondary transmitter to primary receiver channel. Moreover, we transform the EE problem into semidefinite programming (SDP) problem to guarantee a global optimal solution. Analytical results are provided for scheme 1, whereas, the problem of scheme 2 is written in a standard SDP form, which can be solved in polynomial time. Numerical results show that the optimal beamformer which maximizes the SINR (scheme 1) is not the one to maximize the EE metric (scheme 2). Furthermore, we show that the proposed schemes outperform the same schemes that do not utilize the sensing information. Note that in order to meet the pages limit, we shorten this version of the paper by removing many details, proofs and references. We have uploaded a longer and full version of this paper in [1].

Unlike the work in [4], which analyzes the EE metric without considering a PU protection constraint and no utilization of the sensing information, our work protects the PU and utilizes the sensing information.

This paper is organized as follows. Section II describes the system model with some related background on PU blind multi-antenna sensing approach. Section III presents the common constraints and preliminaries for both targeted optimization problems. Section IV tackles the problem of maximizing SINR problem with the corresponding constraints. In section $\mathrm{V}$ we maximize the EE problem with the corresponding constraints. Finally, some numerical results are presented in section VI.

\section{System Model ANd Related BACKGRound}

\section{A. System Model}

In this design, we consider the sensing information effect on the following cases,

1) Maximizing the SU's SINR under both a peak power constraint and an interference constraint.

2) Maximizing the SU's EE quantity (defined in section V) under peak power, interference, and minimum rate constraints.

Figure 1 shows the system model under the assumption of MIMO channels for ST-SR, PT-SR, and ST-PR, where ST and SR are the secondary transmitter and receiver, respectively. PT and PR are the primary transmitter and receiver, respectively. The SU sensor assumed to be located at the SR, while the sensing information is exchanged between SR and ST through a low-rate error free feedback channel without considerable delay. We assume that ST has $N_{t}$ antennas, SR has $N_{r}$ antennas, PT has $M_{t}$ antennas, and PR has $M_{r}$ antennas. 
The channels depicted in Fig. 1 are as follows, $\mathbf{H}_{p s} \in \mathbb{C}^{N_{r} \times M_{t}}$ is the channel between PT-SR, $\mathbf{H}_{s p} \in \mathbb{C}^{M_{r} \times N_{t}}$ is the channel between ST-PR, and $\mathbf{H}_{s} \in \mathbb{C}^{N_{r} \times N_{t}}$ is the channel between SR and ST. These channels are independent of each other and identically distributed (I.I.D), with entries of each matrix following a circularly symmetric complex Gaussian random variables with zero mean and unit variance. The CSI of $\mathbf{H}_{s}$ is assumed to be known and shared between ST and SR through the feedback channel, while the SU has no knowledge of $\mathbf{H}_{s p}$ neither at the ST nor at the SR. The SR decodes the interference from the PT as noise and no successive interference cancellation scheme is implemented.

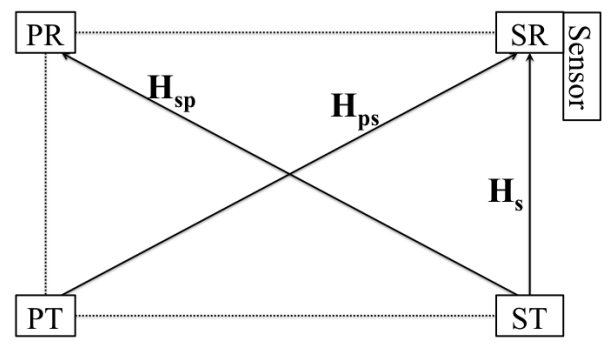

Fig. 1. System Model.

\section{B. Sensing Background}

In this section, we outline our sensing approach used in the proposed system. This approach is based on blind combining, no knowledge of $\mathbf{H}_{p s}$ is utilized, the PU signal at the multi-antenna SU sensor then applying the energy detector to the output. This sensing scheme have appeared in [6], we recall it here for self-contentedness of the paper. Moreover, we provide analytical results which have not been mentioned in [6], but are necessary to characterize the sensor error performance, and essential to obtain the interference threshold of the beamforming weights.

When the primary user is transmitting, the SU sensor observes a signal $\mathbf{Y}=\left[\mathbf{y}_{1}, \mathbf{y}_{2}, \ldots, \mathbf{y}_{N_{s}}\right]$, where $\mathbf{y}_{i} \in \mathbb{C}^{N_{r \times 1}}, N_{s}$ is the number of sensing samples, and $\mathbf{y}_{i}$ is given by,

$$
\mathbf{y}_{i}=\mathbf{H}_{p s, i} \mathbf{x}_{p, i}+\mathbf{n}_{s, i} \quad i=1, \ldots, N_{s}
$$

where $i$ is the sampling index, $\mathbf{x}_{p, i}$ is the PT transmitted signal at sample $i$, the vector $\mathbf{n}_{s, i}$ represents the additive white Gaussian noise (AWGN) at SR and sample $i$ with zero mean and variance $\frac{N_{0}}{2}$ per dimension. The SU sensor then computes the Wishart matrix, $\mathbf{R}_{\mathbf{y}}=\mathbf{Y} \mathbf{Y}^{\dagger}$, where the upper script $(.)^{\dagger}$ designates the conjugate transpose of its argument. Using the Eigen-Value-Decomposition (EVD) method, the following is true, $\mathbf{R}_{\mathbf{y}} \mathbf{V}=\mathbf{V} \boldsymbol{\Lambda}$, where $\boldsymbol{\Lambda}$ is a diagonal matrix with $v_{r}, \ldots, v_{2}, v_{1}$ are the elements in the diagonal. The diagonal elements, $v_{r} \geq \cdots \geq v_{2} \geq v_{1}$, are the Wishart matrix's eigenvalues, where $r=\min \left(N_{r}, N_{s}\right)$. The matrix $\mathbf{V}=\left[\mathbf{v}_{r}, \ldots, \mathbf{v}_{1}\right]$, and its column vectors $\mathbf{v}_{\mathbf{r}}, \ldots, \mathbf{v}_{\mathbf{1}}$ are the corresponding eigenvectors. To realize the maximum SNR, we use the eigenvector that corresponds to the largest eigenvalue as the combining weight $\mathbf{w}$ for the SU sensor antennas, $\mathbf{w}=\mathbf{v}_{\mathbf{r}}$.

The output of the combiner is expressed as:

$$
\mathbf{y}_{\text {evd }}=\mathbf{w}^{\dagger} \mathbf{Y} .
$$

where $\mathbf{y}_{\text {evd }} \in \mathbb{C}^{1 \times N_{s}}$. Note that

$$
\mathbf{y}_{e v d} \mathbf{y}_{e v d}^{\dagger}=\mathbf{w}^{\dagger} \mathbf{R}_{\mathbf{y}} \mathbf{w}=\mathbf{v}_{\mathbf{r}}^{\dagger} v_{r} \mathbf{v}_{\mathbf{r}}=v_{r}\left\|\mathbf{v}_{\mathbf{r}}\right\|^{2} \stackrel{(a)}{=} v_{r}
$$

where (a) is obtained because the norm of the combining weights $\mathbf{v}_{\mathbf{r}}$ is unity. This confirms the fact that the EVD scheme uses combining weights that beamforms the sensing antennas to the largest eigenmode of the SR-PT channel. We apply the conventional energy detector to the combiner output signal $\mathbf{y}_{\text {evd }}$. The output of the energy detector before decision making step is as follows,

$$
\zeta\left(N_{s}\right)=\frac{1}{N_{s}} \sum_{i=1}^{N_{s}}\left|\mathbf{y}_{\text {evd }}(i)\right|^{2}=\frac{1}{N_{s}} \mathbf{y}_{\text {evd }} \mathbf{y}_{\text {evd }}^{\dagger}=\frac{1}{N_{s}} v_{r} .
$$

The distribution of $\zeta\left(N_{s}\right)$ is (up to a scaling) similar to the largest eigenvalue distribution [1] with a probability density function (PDF) as follows,

$$
f\left(v_{r}\right)=\frac{\left|\boldsymbol{\Psi}_{c}\left(v_{r}\right)\right| \operatorname{tr}\left(\mathbf{\Psi}_{c}^{-1}\left(v_{r}\right) \boldsymbol{\Phi}_{c}\left(v_{r}\right)\right) U\left(v_{r}\right)}{\prod_{k=1}^{r} \Gamma(t-k+1) \Gamma(r-k+1)}
$$

where $r=\min \left(N_{r}, N_{s}\right)$ and $t=\max \left(N_{r}, N_{s}\right)$, the function $U($.$) is a unit step function. The scalar v_{r}$ is the maximum eigenvalue of the normalized Wishart matrix $\boldsymbol{\Sigma}^{-\mathbf{1}} \mathbf{R}_{\mathbf{y}}$, where $\boldsymbol{\Sigma}$ is the covariance matrix expressed as $\boldsymbol{\Sigma}=$ $\mathbb{E}_{\mathbf{x}_{p, i}, \mathbf{H}_{p s, i}}\left\{\left(\mathbf{H}_{p s, i} \mathbf{x}_{p, i}+\mathbf{n}_{s, i}\right)\left(\mathbf{H}_{p s, i} \mathbf{x}_{p, i}+\mathbf{n}_{s, i}\right)^{\dagger}\right\}$, and $\mathbb{E}\{$.$\} is$ the expected value of its argument. Since we consider i.i.d. Rayleigh fading central channel and $\mathbf{x}_{p, i}$ is an i.i.d. random variable, then the covariance matrix is expressed as $\boldsymbol{\Sigma}=\sigma \mathbf{I}$, where $\sigma$ is the variance of the received PU signal at SU sensor. The function $\Gamma($.) is the gamma function [1], the function $\boldsymbol{\Phi}_{c}\left(v_{r}\right)$ is an $r \times r$ matrix whose entries are given by, $\left[\boldsymbol{\Phi}_{c}\left(v_{r}\right)\right]_{i, j}=v_{r}^{t-r+i+j-2} e^{-v_{r}}$, and $\left[\boldsymbol{\Psi}_{c}(x)\right]_{i, j}=\gamma(t-r+i+j-1, x), \forall i, j=1, \ldots, r$, where, the $\gamma($.$) is the incomplete gamma function.$

$$
\begin{aligned}
& \text { Reformulating (4) in terms of PU existence such that, } \\
& \zeta\left(N_{s}\right)= \begin{cases}\zeta_{1}\left(N_{s}\right)=\frac{1}{N_{s}} \sum_{i=1}^{N_{s}}\left|\mathbf{w}^{\dagger}\left[\mathbf{H}_{p s, i} \mathbf{x}_{p, i}+\mathbf{n}_{s, i}\right]\right|^{2} & ; \mathcal{H}_{1} \\
\zeta_{0}\left(N_{s}\right)=\frac{1}{N_{s}} \sum_{i=1}^{N_{s}}\left|\mathbf{w}^{\dagger} \mathbf{n}_{s, i}\right|^{2} & ; \mathcal{H}_{0}\end{cases}
\end{aligned}
$$

where $\mathcal{H}_{1}$ and $\mathcal{H}_{0}$ are the hypotheses at which the primary user is active and idle, respectively. It follows that $f_{1}\left(v_{r}\right)$ and $f_{0}\left(v_{r}\right)$ are the corresponding PDF of $\zeta_{1}\left(N_{s}\right)$ and $\zeta_{0}\left(N_{s}\right)$, respectively. Based on the two hypotheses in (6) we divide the output of the SU sensor into four regions as follows,

$$
\begin{array}{llll}
\mathcal{R}_{11}: & \zeta\left(N_{s}\right) \geq \gamma_{t h} & \text { under } \mathcal{H}_{1} \text { (Correct detection) } \\
\mathcal{R}_{10}: & \zeta\left(N_{s}\right) \geq \gamma_{t h} \text { under } \mathcal{H}_{0} \text { (False alarm) } \\
\mathcal{R}_{01}: & \zeta\left(N_{s}\right) \leq \gamma_{t h} \text { under } \mathcal{H}_{1} \text { (Miss detection) } \\
\mathcal{R}_{00}: & \zeta\left(N_{s}\right) \leq \gamma_{t h} \text { under } \mathcal{H}_{0} \text { (Correct event) }
\end{array}
$$

where $\gamma_{t h}$ is the detection threshold. For the sake of the numerical simulation notation let us note the probability of the region as, $\operatorname{Pr}\left\{\mathcal{R}_{11}\right\}=P_{D}, \operatorname{Pr}\left\{\mathcal{R}_{10}\right\}=P_{F A}$. Our purpose of using the sensing is to link the power allocation scheme to the sensing results. In other words, based on the regions defined in (7) we define, in Sec. IV, different thresholds for the interference caused to the PR by the ST. In order to achieve more protection for the PR, we join the regions in (7) into only two events. The first event (called $\mathcal{A}$ ) occurs when the SU sensor declare the existence of PU. We define the probability of event $\mathcal{A}$ as follows (knowing that $\operatorname{Pr}\left\{\mathcal{H}_{1}\right\}+\operatorname{Pr}\left\{\mathcal{H}_{0}\right\}=1$ ),

$$
\begin{aligned}
& \operatorname{Pr}\{\mathcal{A}\}=\operatorname{Pr}\left\{\mathcal{R}_{11} \cup \mathcal{R}_{10}\right\}= \\
& \operatorname{Pr}\left\{\zeta\left(N_{s}\right) \geq \gamma_{t h} \mid \mathcal{H}_{1}\right\} \operatorname{Pr}\left\{\mathcal{H}_{1}\right\}+\operatorname{Pr}\left\{\zeta\left(N_{s}\right) \geq \gamma_{t h} \mid \mathcal{H}_{0}\right\} \operatorname{Pr}\left\{\mathcal{H}_{0}\right\} \\
& =1-F_{1}\left(v_{r}\right) \operatorname{Pr}\left\{\mathcal{H}_{1}\right\}-F_{0}\left(v_{r}\right) \operatorname{Pr}\left\{\mathcal{H}_{0}\right\}
\end{aligned}
$$

where the functions $F_{0}\left(v_{r}\right)$ and $F_{1}\left(v_{r}\right)$ is the corresponding cumulative density function (CDF) under hypotheses $\mathcal{H}_{0}$ and $\mathcal{H}_{0}$, respectively. Since $\mathcal{A}^{c}$ is the event where the SU sensor declares that $\mathrm{PU}$ is idle, then, $\operatorname{Pr}\left\{\mathcal{A}^{c}\right\}=1-\operatorname{Pr}\{\mathcal{A}\}$. 


\section{Preliminaries \& Common Constraints}

In this section, we present common constraints that are shared by the maximization of SINR and EE problems. The received signal at SR is expressed as,

$$
\mathbf{y}=\mathbf{H}_{p s} \mathbf{x}_{p}+\mathbf{H}_{s} \mathbf{u}_{s}+\mathbf{n}_{s}
$$

where $\mathbf{y}=\left[y_{1}, \ldots, y_{N_{r}}\right]$. The vector $\mathbf{u}_{s}$ is the pre-coding vectors of the ST (the secondary data $\mathbf{x}_{s}$ is included in $\mathbf{u}_{s}$ ). Note that, $\left\|\mathbf{x}_{p}\right\|^{2}=$ $P_{p}$ and $\left\|\mathbf{u}_{s}\right\|^{2}=P_{\max }$ are the transmission power for both PT and ST, respectively. The received signal after multiplying it by the receiving beamforming vector is,

$$
r=\mathbf{v}_{s}^{\dagger} \mathbf{y}
$$

where $\mathbf{v}_{s}$ is the post-coding vector at SR, note that $\left\|\mathbf{v}_{s}\right\|^{2}=1$.

The first constraint, which is common between both SINR and EE problem, is the peak power constraint $\left(\mathfrak{C}_{1}\right)$ on the $\mathrm{ST}$ transmission power, which is expressed as,

$$
\mathfrak{C}_{1}:\left\|\mathbf{u}_{s}\right\|^{2} \leq P_{\max }
$$

where $P_{\max }$ is the maximum peak power on ST.

The second common constraint is the statistical interference constraint $\left(\mathfrak{C}_{2}\right)$ on the $\mathrm{ST}$ interference power affecting the PR, expressed as below,

$$
\mathfrak{C}_{2}: \operatorname{Pr}_{\mathbf{v}_{p}, \mathbf{H}_{s p}}\left\{\left|\mathbf{v}_{p}^{\dagger} \mathbf{H}_{s p} \mathbf{u}_{s}\right|^{2} \leq P\right\} \geq 1-\delta
$$

where the vector $\mathbf{v}_{p}$ is the PR weighting vector, $\left\|\mathbf{v}_{p}\right\|^{2}=1$, the constant $P$ is the interference threshold toward the PR which is decided based on the sensing information, and $\delta$ is the probability value with which the ST is allowed to violate the interference threshold $P$.

\section{MAXIMIZING SINR}

In this section, we formulate the problem of maximizing SINR utilizing the sensing information. Based on the received signal in (10), the corresponding secondary user SINR is [7],

$$
\operatorname{SINR}=\frac{\left(\mathbf{v}_{s}^{\dagger} \mathbf{H}_{s} \mathbf{u}_{s}\right)^{\dagger}\left(\mathbf{v}_{s}^{\dagger} \mathbf{H}_{s} \mathbf{u}_{\mathbf{s}}\right)}{\left(\mathbf{v}_{s}^{\dagger} \mathbf{H}_{s p} \mathbf{x}_{p}\right)^{\dagger}\left(\mathbf{v}_{s}^{\dagger} \mathbf{H}_{s p} \mathbf{x}_{p}\right)+N_{0}}
$$

Note that our choice of this SINR formula follows from several facts. This expression is an achievable SINR, since there is interference from the PU and the MIMO system is not a point-to-point. Moreover, We consider a similar SINR formula in the EE maximization problem for the ease of transformation to SDP problem. Therefore, in order to preserve fairness in comparison, we use the same SINR formula in maximizing the rate (note that, in this paper, because of the space limitation we could not insert this comparison between EE and SINR performance). It is important to note that maximizing the corresponding rate metric, $R=\log (1+\mathrm{SINR})$, is equivalent to the maximization of the SINR metric, since the logarithm is a monotonically increasing function. The maximum SINR optimization problem is thus formulated as follows,

$$
\begin{aligned}
P_{1}: \max _{\mathbf{u}_{s}} & \text { SINR } \\
\text { s.t. } & \mathfrak{C}_{1}:\left\|\mathbf{u}_{s}\right\|^{2} \leq P_{\max } \\
& \mathfrak{C}_{2}: \operatorname{Pr}_{\mathbf{v}_{p}, \mathbf{H}_{s p}}\left\{\left|\mathbf{v}_{p}^{\dagger} \mathbf{H}_{s p} \mathbf{u}_{s}\right|^{2} \leq P\right\} \geq 1-\delta
\end{aligned}
$$

After incorporating the sensing information in $(14 \mathrm{c}), \mathfrak{C}_{2}$ is reformulated as follows,

$$
\begin{aligned}
\mathfrak{C}_{2}= & \operatorname{Pr}_{\mathbf{v}_{p}, \mathbf{H}_{s p}}\left\{\left|\mathbf{v}_{p}^{\dagger} \mathbf{H}_{s p} \mathbf{u}_{s}\right|^{2} \leq P \mid \mathcal{A}\right\} \operatorname{Pr}\{\mathcal{A}\}+ \\
& \operatorname{Pr}_{\mathbf{v}_{p}, \mathbf{H}_{s p}}\left\{\left|\mathbf{v}_{p}^{\dagger} \mathbf{H}_{s p} \mathbf{u}_{s}\right|^{2} \leq P \mid \mathcal{A}^{c}\right\} \operatorname{Pr}\left\{\mathcal{A}^{c}\right\} \geq 1-\delta
\end{aligned}
$$

Considering our sensing scheme we split $P$ depending on the occurrence of $\mathcal{A}$ or $\mathcal{A}^{c}$, as follows,

$$
P=\left\{\begin{array}{ll}
Q_{\text {int }} & ; \mathcal{A} \\
P_{\text {ave }} & ; \mathcal{A}^{c}
\end{array} .\right.
$$

Our objective is to tune the interference threshold $P$ depending on the sensing information. Intuitively, if the primary is active, then $P$ is set to the lowest value, otherwise, $P$ can be arbitrary high. It is then clear that $Q_{\text {int }} \leq P_{\text {ave }}$.

Now we begin solving constraint $\mathfrak{C}_{2}$ which can be tackled as follows. Note that $\mathbf{v}_{p}$ and $\mathbf{H}_{s p}$ are independent upon conditioning on $\mathbf{v}_{p}$. Then, the quantity $\mathbf{v}_{p}^{\dagger} \mathbf{H}_{s p} \mathbf{u}_{s}$ is a complex Gaussian random variable with mean 0 and variance $\left\|\mathbf{u}_{s}\right\|^{2}$, recall that $\left\|\mathbf{v}_{p}\right\|^{2}=1$. Note that the conditional distribution of the quantity $\mathbf{v}_{p}^{\dagger} \mathbf{H}_{s p} \mathbf{u}_{s}$ is independent of $\mathbf{v}_{p}$. Therefore, unconditionally $\mathbf{v}_{p}^{\dagger} \mathbf{H}_{s p} \mathbf{u}_{s} \sim \mathcal{C N}\left(0,\left\|\mathbf{u}_{s}\right\|^{2}\right)$. It follows that $\left|\mathbf{v}_{p}^{\dagger} \mathbf{H}_{s p} \mathbf{u}_{s}\right|^{2}$ is exponentially distributed, with parameter $\frac{1}{\left\|\mathbf{u}_{s}\right\|^{2}}$. Therefore, (15) is expressed as,

$$
1-e^{-\left(\frac{Q_{\text {int }}}{\left\|\mathbf{u}_{s}\right\|^{2}}\right)} \operatorname{Pr}\{\mathcal{A}\}-e^{-\left(\frac{P a v e}{\left\|\mathbf{u}_{s}\right\|^{2}}\right)} \operatorname{Pr}\left\{\mathcal{A}^{c}\right\} \geq 1-\delta,
$$

Let us call the left hand side of (17) as $F_{p}\left(\left\|\mathbf{u}_{s}\right\|^{2}\right)$, which is an invertible function [1]. We can rewrite (17) as follows,

$$
\left\|\mathbf{u}_{s}\right\|^{2} \leq F_{p}^{-1}(1-\delta)
$$

where, $F_{p}^{-1}(1-\delta)$ is the numerically obtained zero of (17). For the ease of notation, we introduce a new variable $\beta=$ $\min \left(P_{\max }, F_{p}^{-1}(1-\delta)\right)$. Both constraints of problem (14) $\mathfrak{C}_{1}$ and $\mathfrak{C}_{2}$ are reduced to,

$$
\left\|\mathbf{u}_{s}\right\|^{2} \leq \beta
$$

From (14a), the beamforming receiving vector that optimally maximizes the SINR at the SR, is expressed as $\mathbf{v}_{s}=\mu_{s} \boldsymbol{\Phi}^{-1} \mathbf{H}_{s} \mathbf{u}_{s}$, where, $\boldsymbol{\Phi}=\left(\mathbf{H}_{p s} \mathbf{x}_{p}\right)\left(\mathbf{H}_{p s} \mathbf{x}_{p}\right)^{\dagger}+N_{0} \mathbf{I}$, and $\mu_{s}=\frac{1}{\left\|\boldsymbol{\Phi}^{-1} \mathbf{H}_{s} \mathbf{u}_{s}\right\|}$ [1]. Note that to obtain $\boldsymbol{\Phi}$ we do not need to know $\mathbf{H}_{p s}$ or $\mathbf{x}_{p}$, since we are concerned with the interference plus noise term as a whole quantity, and this can be measured frequently when ST stops transmitting.

Utilizing the definition of $\boldsymbol{\Phi}$ and $\mathbf{v}_{s}$ the SINR in (13) can be reformulated as follows,

$$
\begin{aligned}
\operatorname{SINR}= & \frac{\mathbf{u}_{s}^{\dagger} \mathbf{H}_{s}^{\dagger} \mathbf{v} \mathbf{v}_{s}^{\dagger} \mathbf{H}_{s} \mathbf{u}_{s}}{\operatorname{Tr}\left[\mathbf{v}_{s} \mathbf{v}_{s}^{\dagger} \mathbf{H}_{p s} \mathbf{x}_{p} \mathbf{x}_{p}^{\dagger} \mathbf{H}_{p s}^{\dagger}\right]+N_{0}}=\frac{\mathbf{u}_{s}^{\dagger} \mathbf{H}_{s}^{\dagger} \mathbf{v}_{s} \mathbf{v}_{s}^{\dagger} \mathbf{H}_{s} \mathbf{u}_{s}}{\mathbf{v}_{s}^{\dagger} \mathbf{\Phi} \mathbf{v}_{s}} \\
& =\mathbf{u}_{s}^{\dagger} \mathbf{B} \mathbf{u}_{s},
\end{aligned}
$$

where $\mathbf{B}=\mathbf{H}_{s}^{\dagger} \boldsymbol{\Phi}^{-1} \mathbf{H}_{s}$.

It follows that problem (14) becomes,

$$
\begin{array}{ll}
P_{1}^{\prime}: \operatorname{Max} & \mathbf{u}_{s}^{\dagger} \mathbf{B} \mathbf{u}_{s} \\
& \left\|\mathbf{u}_{s}\right\|^{2} \leq \beta
\end{array}
$$

Problem $P_{1}^{\prime}$ is known as the eigenvalue problem. Its solution can be derived by finding the largest eigenvalue of matrix $\mathbf{B}$ and its associated eigenvector, $\mathbf{b}_{m}$. The solution of $P_{1}^{\prime}$ is expressed exactly as $\sqrt{\beta} \mathbf{b}_{m}$. For more details check [1].

\section{Maximizing EE}

In this section, we maximize the EE metric subject to minimum rate, peak power, and interference constraints. The received signal at the SR is defined as in (9) or after post-coding as in (10). Using the expression of SINR in (13), the SU rate expression is $R=\log (1+\mathrm{SINR})$, we define the EE as follows [1],

$$
\mathrm{EE}=\frac{R}{P_{s}}=\frac{\log (1+\mathrm{SINR})}{\left\|\mathbf{u}_{s}\right\|^{2}}
$$


The EE maximization problem is formulated as follows,

$$
\begin{array}{cl}
P_{2}: \max _{\mathbf{u}_{s}} & \mathrm{EE} \\
\text { s.t. } & \left\|\mathbf{u}_{s}\right\|^{2} \leq P_{\max } \\
& \operatorname{Pr}_{\mathbf{v}_{p}, \mathbf{H}_{s p}\left\{\left|\mathbf{v}_{p}^{\dagger} \mathbf{H}_{s p} \mathbf{u}_{s}\right|^{2} \leq P\right\} \geq 1-\delta} \\
& \log (1+\operatorname{SINR}) \geq R_{\text {min }}
\end{array}
$$

where the constant $R_{\text {min }}$ is the minimum acceptable rate threshold, and the newly introduced constraint (23d) is a minimum rate constraint. Note that problem $P_{2}$ is a fractional problem, which is not an obvious concave problem. Therefore, we transform this problem into an SDP problem. Hereafter, we use the definition of equivalency between two problems as in [1], (i.e., "Two problems are equivalent if from a solution of one, a solution of the other is readily found, and vice versa").

The first step of the SDP transformation is to introduce a new optimization variable $t$ that changes the objective function (23a) into a constraint, $\frac{\log \left(1+\gamma_{s}\right)}{\operatorname{Tr}[\mathbf{S}]} \geq \frac{1}{t}$, while minimizing a different objective function, i.e. " $t$ ", [1]. Furthermore, we enforce a new equality constraint as $\operatorname{SINR}=\mathbf{u}_{s} \mathbf{B} \mathbf{u}_{s}^{\dagger}=\operatorname{Tr}[\mathbf{B S}]=\gamma_{s}$, where $\mathbf{S}=\mathbf{u}_{s} \mathbf{u}_{s}^{\dagger}$. Then, problem $P_{2}$ becomes equivalent to,

$$
\begin{array}{rl}
P_{2}^{\prime}: \min _{\mathbf{S}, t} & t \\
\text { s.t } & \frac{\log \left(1+\gamma_{s}\right)}{\operatorname{Tr}[\mathbf{S}]} \geq \frac{1}{t} \\
& \operatorname{Tr}[\mathbf{B S}]=\gamma_{s} \\
& \operatorname{Tr}[\mathbf{S}] \leq \beta \\
& \operatorname{Tr}[\mathbf{B S}]-\left(\exp \left(R_{\text {min }}\right)-1\right) \geq 0 \\
& \mathbf{S} \succeq 0 \\
& \operatorname{rank}[\mathbf{S}]=1,
\end{array}
$$

Note that constraints [(23b), (23c)], and (23d) are equivalent to constraints [(24d)], and (24e), respectively. Constraint $(24 \mathrm{~g})$ is to guarantee that the optimization variable $\mathbf{S}$ can be written as an outer product of a vector, i.e. $\mathbf{S}=\mathbf{u}_{s} \mathbf{u}_{s}^{\dagger}$. The equivalence between $P_{2}$ and $P_{2}^{\prime}$ is explained in details in [1].

Problem $P_{2}^{\prime}$, without considering constraint $(24 \mathrm{~g})$, is an SDP problem but not in the standard form. We transform $P_{2}^{\prime}$ into a standard form, leaving the rank constraint to a later step. This can be done by using the fact that $\operatorname{Tr}[\mathbf{A B}]=\operatorname{vec}\left(\mathbf{A}^{T}\right)^{T} \operatorname{vec}(\mathbf{B})$, where $\operatorname{vec}($.$) is the vectorization operator which converts the matrix into$ a column vector by stacking the columns of the matrix on top of one another. Furthermore, we introduce dummy variables for the inequality constraints $(24 \mathrm{~b}),(24 \mathrm{~d}$, and $(24 \mathrm{e})$ which change them into equality constraints (25b), (25d), and (25e), respectively, with the corresponding three dummy variables $r_{1}, r_{2}$, and $r_{3}$. Then, $P_{2}^{\prime}$ becomes,

$$
\begin{array}{llr}
P_{2}^{\prime \prime}: \min _{\mathbf{S}, t, r_{1}, r_{2}, r_{3}} & t \\
\text { s.t } & t \log \left(1+\gamma_{s}\right)-\operatorname{vec}(\mathbf{I})^{T} \operatorname{vec}(\mathbf{S})-r_{1}=0 \\
& \operatorname{vec}\left(\mathbf{B}^{T}\right)^{T} \operatorname{vec}(\mathbf{S})=\gamma_{s} & (25 \mathrm{a}) \\
& \beta-\operatorname{vec}(\mathbf{I})^{T} \operatorname{vec}(\mathbf{S})-r_{2}=0 \\
& \operatorname{vec}\left(\mathbf{B}^{T}\right)^{T} \operatorname{vec}(\mathbf{S})-\left(\exp \left(R_{\text {min }}\right)-1\right)-r_{3}=0 \\
& \mathbf{S} \succeq 0 \\
& \left.r_{i} \geq 0 \quad i=1,2,3 \mathrm{c}\right) \\
& \operatorname{rank}[\mathbf{S}]=1
\end{array}
$$

Finally, by defining a new optimization variable $\mathbf{x}=$ $\left[\operatorname{vec}(\mathbf{S})^{T}, t, r_{1}, r_{2}, r_{3}\right]^{T}$, new vectors $\mathbf{b}=\left[\gamma_{s}, 0,-\beta, e^{R_{\text {min }}}-1\right]^{T}$ and $\mathbf{c}=\left[\mathbf{0}_{N_{t}^{2} X 1}^{T}, 1,0,0,0\right]^{T}$, and a new matrix

$$
\mathbf{A}=\left[\begin{array}{ccccc}
\operatorname{vec}\left(\mathbf{B}^{T}\right)^{T} & 0 & 0 & 0 & 0 \\
-\operatorname{vec}(\mathbf{I})^{T} & \log \left(1+\gamma_{s}\right) & -1 & 0 & 0 \\
-\operatorname{vec}(\mathbf{I})^{T} & 0 & 0 & -1 & 0 \\
\operatorname{vec}\left(\mathbf{B}^{T}\right)^{T} & 0 & 0 & 0 & -1
\end{array}\right]
$$

it is possible to reformulate $P_{2}^{\prime \prime}(25)$ into standard SDP form as follows,

$$
\begin{aligned}
P_{2}^{\prime \prime \prime}: \min _{\mathbf{x}} & \mathbf{c}^{T} \mathbf{x} \\
\text { s.t. } & A \mathbf{x}=\mathbf{b} \\
& {[\mathbf{x}]_{k} \geq 0, k=N_{t}^{2}+1, \ldots, N_{t}^{2}+4 . } \\
& \operatorname{mat}\left([\mathbf{x}]_{N_{t}^{2}}^{1}\right)_{N_{t}} \succeq 0
\end{aligned}
$$

where, the operator $[\mathbf{x}]_{k}$ represents the $k^{\text {th }}$ element of vector $\mathbf{x}$, the operator $[\mathbf{x}]_{N_{t}^{2}}^{1}$ represents the elements from index 1 to index $N_{t}^{2}$ of vector $\mathbf{x}$. The term $[\mathbf{x}]_{k} \geq 0$ is an element wise inequality, and the function mat $\left([\mathbf{x}]_{N_{t}^{2}}^{1}\right)_{N_{t}}$ transforms the vector $\mathbf{x}$ to matrix by aligning the first $N_{t}$ elements of $\mathbf{x}$ as the first column in the matrix, and so on. The above problem is recognize as an SDP problem that can be solved by the interior-point method, or other available modules (CVX Matlab [1]). It is observed that the rank-1 constraint is dropped in $P_{2}^{\prime \prime \prime}$, which means that problem $P_{2}^{\prime \prime \prime}$ is a relaxed version of $P_{2}^{\prime \prime}$. There are several ways to find the optimal solution of $P_{2}^{\prime \prime}$ from the optimal solution of $P_{2}^{\prime \prime \prime}$, as follows. One way is to obtain the optimal solution of $P_{2}^{\prime \prime \prime}, \mathbf{x}^{*}$, and check the rank-1 constraint as follows, $\operatorname{rank}\left[\operatorname{mat}\left(\left[\mathbf{x}^{*}\right]_{N_{t}^{2}}^{1}\right)_{N_{t}}\right]=1$. In case the constraint is satisfied then $\mathrm{x}^{*}$ is optimal for both $P_{2}^{\prime \prime}$ and $P_{2}^{\prime \prime \prime}$ otherwise we declare an outage event. Another way of finding the optimal solution of problem $P_{2}^{\prime \prime}$, without checking the rank, is to use some randomization methods explained by Luo and Chang in Chapter 4 of [8].

\section{NUMERICAL RESULTS}

In this section, we evaluate the proposed schemes with sensing information versus the same scheme without sensing information (which is considered as benchmarks for the proposed schemes) through numerical results. We consider the parameters mentioned in Table I to evaluate the schemes. The evaluation is done by checking the SU maximum achievable EE metric (considered in bits/Joule/Hz), the SU rate (in bits/sec/Hz) that corresponds to the maximum SINR, and the outage metric, which is defined as the event where there is no feasible solution of the corresponding optimization problem.

Knowing that the maximum EE is resulted from minimizing the power to rate ratio. While preserving the minimum rate constraint. Therefore, we define the EE outage probability metric, which is the probability of outage that happens because there is no feasible solution that satisfies all the problem's constraints, as follows,

$$
\text { EE Outage }= \begin{cases}0 & ; \text { A feasible solution exists } \\ 1 & ; \text { No feasible solution }\end{cases}
$$

Because of the space restrictions we limit our figure into three.

Figure 2(a), shows the EE CDF of "with sensing" scheme 2 for different values of $Q_{\text {int }}=-20 \mathrm{dBm},-16 \mathrm{dBm},-10 \mathrm{dBm},-6$ $\mathrm{dBm}$. It is observed that increasing the values of $Q_{\text {int }}$ results in improving the EE performance of the system, from $0.3 \mathrm{bits} / \mathrm{Joule} / \mathrm{Hz}$ up to $2 \mathrm{bits} / \mathrm{Joule} / \mathrm{Hz}$. Furthermore, increasing $Q_{\text {int }}$ more than a certain value does not improve the EE performance, such that, the 

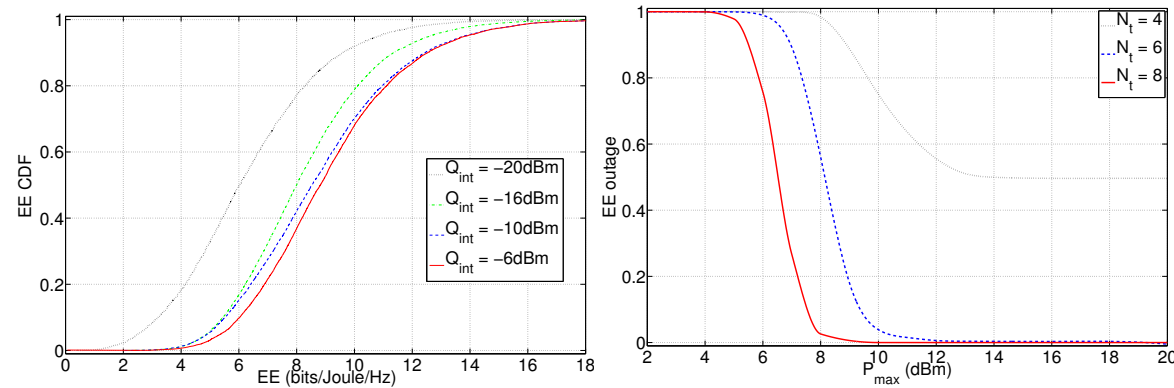

(a) $\mathrm{EE} \mathrm{CDF}$ performance for different $Q_{i n t}=$ (b) EE outage versus $N_{t}=4,6,8$ and $P_{m a x}$ for $-20 \mathrm{dBm},-16 \mathrm{dBm},-10 \mathrm{dBm}$, and $-6 \mathrm{dBm}$. all with sensing schemes.

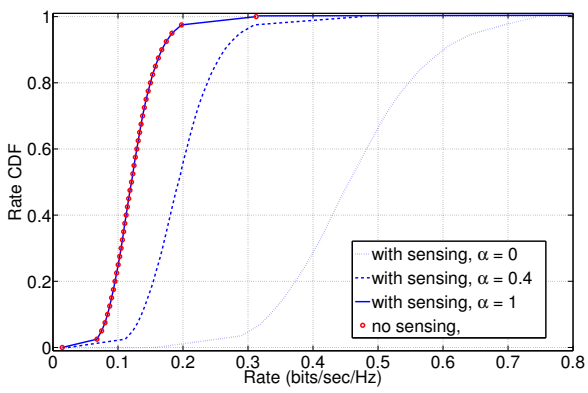

(c) $\mathrm{CDF}$ of the Rate for all schemes with variable $\alpha$.

Fig. 2. All Numerical Results

TABLE I

SIMULATION PARAMETERS.

\begin{tabular}{|l|l|}
\hline Parameter Name & Value \\
\hline Sensing averaging bits & 1000 \\
\hline$P_{\max }$ and $P_{\text {ave }}$ & $27 \mathrm{dBm}$ \\
\hline Maximum PT power $\left(P_{p}\right)$ & $27 \mathrm{dBm}$ \\
\hline \# Monte-Carlo iterations & 10000 \\
\hline Wireless channels & $\begin{array}{l}\text { Rayleigh, } \\
\text { Slow Flat Fading }\end{array}$ \\
\hline$\left(N_{t} ; N_{r}\right)$ & $(4 ; 4)$ \\
\hline$\left(M_{t} ; M_{r}\right)$ & $(2 ; 2)$ \\
\hline$Q_{i n t}$ & $0 \mathrm{dBm}, 3 \mathrm{dBm}$ \\
\hline Minimum Rate $\left(R_{\min }\right)$ & $0.1 \mathrm{bits} / \mathrm{sec} / \mathrm{Hz}$ \\
\hline$\delta$ & 0.1 \\
\hline$\alpha$ & $0.15,0.2$ \\
\hline$P_{D}, P_{F A}$ & $0.9,0.01$ \\
\hline
\end{tabular}

performance of $Q_{\text {int }}=-10 \mathrm{dBm}$ and $Q_{i n t}=-6 \mathrm{dBm}$ is very close.

We show the outage probability of the proposed scheme 2 versus $P_{\max }$ and different number of antennas $N_{t}$ in Fig. 2(b). We note the effect of decreasing the $P_{\max }$ on degrading the outage performance of the proposed scheme for different values of $N_{t}$, namely, $N_{t}=4$, $N_{t}=6$, and $N_{t}=8$. The improvement of EE outage is observed by increasing the number of antenna $N_{t}$. It is observed that at $N_{t}=2$ the outage performance saturates at large values of $P_{\max }$. The performance improvement by change the number of antennas from $N_{t}=4$ to $N_{t}=6$ is at least $2 \mathrm{dBm}$. While the performance improvement by change the number of antennas from $N_{t}=6$ to $N_{t}=8$ is at least $1.8 \mathrm{dBm}$.

Figure 2(c), shows the performance of the proposed and benchmark schemes, under SINR maximization problem, versus $\alpha=0,0.4$, and 1. The parameter $\alpha$ follows a Bernoulli distribution and it expresses the prior probability of PU existence $\alpha=\operatorname{Pr}\left\{\mathcal{H}_{1}\right\}$. As described earlier, $\operatorname{Pr}\{\mathcal{A}\}$ is a function of $\alpha$ and the sensor characteristics $P_{D}$ and $P_{F A}$. It is observed from Fig. 2(c) that for $\alpha=1$ both schemes have exactly the same performance, note that the benchmark scheme is not affected by changing $\alpha$. This result is realistic, since at $\alpha=$ 1 we need to protect the primary at all times, hence the sensing information is not useful. We also note that by increasing $\alpha=0$ to $\alpha=0.4$ the performance degrades about $0.3 \mathrm{bits} / \mathrm{sec} / \mathrm{Hz}$. While it decreases about $0.7 \mathrm{bits} / \mathrm{sec} / \mathrm{Hz}$ when $\alpha$ decreases from $\alpha=0.4$ to $\alpha=1$.

\section{CONCLUSION}

This paper proposed two energy efficient beamforming schemes based on cognitive radio environments. The proposed beamforming schemes utilize the sensing information obtained from the secondary user multi-antenna sensor using a blind combining scheme integrated with an energy detector for primary user signal. The optimization problem of the proposed schemes are not a convex problem. Therefore we convert them to an SDP standard problem to guarantee the global unique solution. We evaluated and analyzed the proposed scheme and the benchmark scheme. The numerical results show an EE performance improvement of the proposed scheme, by increasing $Q_{\text {int }}$, from 0.3 bits $/ \mathrm{Joule} / \mathrm{Hz}$ up to 2 bits/Joule $/ \mathrm{Hz}$.

\section{REFERENCES}

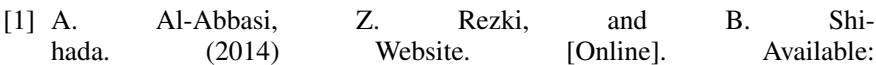
http://www.shihada.com/publications/newchance_13012014_v1_long1.pdf

[2] Z. Hasan, H. Boostanimehr, and V. Bhargava, "Green cellular networks: A survey, some research issues and challenges," Communications Surveys Tutorials, IEEE, vol. 13, no. 4, pp. 524 -540, quarter 2011.

[3] R. S. Prabhu and B. Daneshrad, "An energy-efficient water-filling algorithm for OFDM systems," in IEEE International Conference on Communications (ICC), 2010, 2010, pp. 1-5.

[4] C. Jiang and L. Cimini, "Energy-efficient multiuser MIMO beamforming," in 45th Annual Conference on Information Sciences and Systems (CISS), 2011, pp. 1-5.

[5] S.-J. Kim and G. Giannakis, "Optimal resource allocation for MIMO ad hoc cognitive radio networks," IEEE Transactions on Information Theory, vol. 57, no. 5, pp. 3117-3131, 2011.

[6] A. Al-Abbasi and T. Fujii, "A novel blind diversity detection scheme for multi-antenna cognitive radio spectrum sensing," in Vehicular Technology Conference Fall (VTC 2010-Fall), IEEE 72nd, 2010, pp. 1-5.

[7] L. Zhang, Y.-C. Liang, and Y. Xin, "Joint beamforming and power allocation for multiple access channels in cognitive radio networks," IEEE Journal on Selected Areas in Communications, vol. 26, no. 1, pp. 38-51, 2008.

[8] D. P. Palomar and e. Yonina C. Eldar, Convex Optimization in Signal Processing and Communications. Cambridge University Press, 2009. 